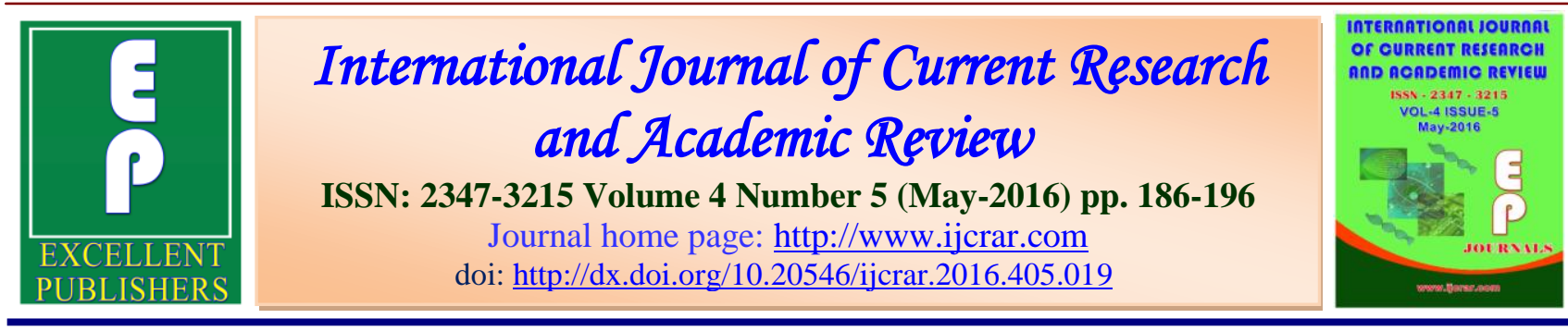

\title{
Microcapsule: A Dosage Form for Drug Delivery in Novel Drug Delivery System
}

\author{
Sonal Salve* and Vaibhav Changediya
}

Dr. Vedprakash Patil Pharmacy College, Auranagbad-431001, India

*Corresponding author

\section{KEYWORDS}

Microencapsulation technology, NDDS, Microcapsule, DNA vaccine.

\begin{abstract}
A B S T R A C T
Microencapsulation is described as a process of enclosing micron sized particles of solids or droplets of liquids or gasses in an inert shell, which in turn isolates and protects them from the external environment as well as control the drug release profile. Microencapsulated particle is having diameter between $3(-) 800 \mu \mathrm{m}$ which differ them from other technologies such as nanotechnology and macroparticle in their morphology and internal structure. This review paper will address the background of micro encapsulation technology, commonly used microencapsulation methods with its advantages and disadvantages and its applications in pharmaceutical field. This article also gives an overview on the general aspects and recent advances in drug-loaded microparticles to improve the efficiency of various medical treatments. This article contains the traditional and the recent pharmaceutical drug delivery system and also established as a unique carrier system for many pharmaceuticals for Novel drug delivery system (NDDS), latest formulation delivery of DNA vaccines prodrug approach, bio degradable and biocompatible material. Other than pharmaceutical microcapsules are widely used in delivery of probiotic, pesticide industry, food technology, beverage and cell immobilization etc.
\end{abstract}

\section{Introduction}

Research on microcapsules is a highly interdisciplinary field that benefits from contributions from various branches of natural sciences and engineering: Already in the thirties biologists, biophysicists and biochemists developed methods that allowed for studies on individual cells, launching the field of microcapsule studies. Research on biological microcapsules remains highly vital and a source of innovation nowadays but has been complemented by research on artificial microcapsule systems. Progress in material sciences allowed production of microcapsules whose structure and composition are controlled on the nanoscale (Tarcha, 1991; Chemical Society Reviews, 2000; Sukhorukov, 2003; Peyratout, 2004; 
Discher et al., 1999; Dinsmore et al., 2002).

To achieve this goal similar mechanisms to those occurring in natural systems, like supramolecular self-assembly, were exploited and most artificial microcapsule systems are to some degree biomimetic. Potential applications of such artificial systems are in most cases linked to encapsulation and release or protection of agents like medicine, fragrances, dyes or flavour additives. Microcapsules can thus be found in fields as diverse as medicine, cosmetics, food design or coating of textiles/paper to mention just a few examples.

\section{Core Material}

The core material is the specific material to be coated, can be liquid or solid in nature. The composition of the core material can be varied as the liquid core can include dispersed and/or dissolved material. The solid core can be mixture of active constituents, stabilizers, diluents, excipients and release-rate retardants or accelerators.

\section{Coating Material}

The selection of appropriate coating material decides the physical and chemical properties of the resultant product. While selecting a polymer the product requirements ie. stabilization, reduced volatility, release characteristics, environmental conditions, etc. should be taken into consideration. The polymer should be capable of forming a film that is cohesive with the core material. It should be chemically compatible, nonreactive with the core material and provide the desired coating properties

\section{Rationale for Microencapsulation}

To attain the sustained or prolonged release of the drug.
For masking the organoleptic properties like taste and odour of many drugs and thus improve patient compliance.

The liquid drugs can be converted into a free flowing powder.

The drugs which are sensitive to moisture light and oxygen can be protected by microencapsulation.

\section{Mechanism and Kinetics of Drug Release}

Major mechanisms of drug release from microcapsules (Brazel, 2000) include diffusion, dissolution, osmosis and erosion:

\section{Diffusion}

The most common mechanism of drug release (core material) in which the dissolution fluid penetrates the shell then the core material comes into the contact with the dissolution fluid and leak out through the interstitial channels or pores (Korsmeyer et $a l .$, 1983). The drug release depends on the rate of drug dissolution in the dissolution fluid, rate of penetration of dissolution fluid to the microcapsules and rate at which the dissolved drug escapes from the microcapsule (Gunder et al., 1995).

\section{Dissolution}

The release rate of the drug from the microcapsule depends on the dissolution rate of polymer coat, when the coat is soluble in the dissolution fluid (Korsmeyer et al., 1983).The solubility in the dissolution fluid and thickness of coat influence the release rate.

\section{Osmosis}

Another method of drug release is through osmosis. The essential requirement of 
osmosis is semi permeable membrane and in microcapsule polymer coat serve the purpose. As the process progress an osmotic pressure is created between the outside and the inside membrane of microcapsule which result in release of drug through small pores. (Lachman et al.; Guo, 1984)

\section{Techniques to Manufacture Microcapsules}

Preparation of microspheres should satisfy certain criteria:

The ability to incorporate reasonably high concentrations of the drug.

Stability of the preparation after synthesis with a clinically acceptable shelf life.

Controlled particle size and dispersability in aqueous vehicles for injection.

Release of active reagent with a good control over a wide time scale.

Biocompatibility with a controllable biodegradability and Susceptibility to chemical modification

\section{Physical Method}

\section{Air-suspension Coating}

Microencapsulation by air suspension technique consist of the dispersing of solid, particulate core materials in a supporting air stream and the spray coating on the air suspended particles. Within the coating chamber, particles are suspended on an upward moving air stream. The design of the chamber and its operating parameters effect a recirculating flow of the particles through the coating zone portion of the chamber, where a coating material, usually a polymer solution, is spray applied to the moving particles. During each pass through the coating zone, the core material receives an increment of coating material. The cyclic process is repeated, perhaps several hundred times during processing, depending on the purpose of microencapsulation the coating thickness desired or whether the core material particles are thoroughly encapsulated. The supporting air stream also serves to dry the product while it is being encapsulated. Drying rates are directly related to the volume temperature of the supporting air stream. Air-suspension coating of particles by solutions or melts gives better control and flexibility. The particles are coated while suspended in an upward-moving air stream. They are supported by a perforated plate having different patterns of holes inside and outside a cylindrical insert. Just sufficient air is permitted to rise through the outer annular space to fluidize the settling particles. Most of the rising air (usually heated) flows inside the cylinder, causing the particles to rise rapidly. At the top, as the air stream diverges and slows, they settle back onto the outer bed and move downward to repeat the cycle.

\section{Coacervation and Microencapsulation}

Coacervation is a colloid phenomenon. If one starts with a solution of a colloid in an appropriate solvent, then according to the nature of the colloid, various changes can bring about a reduction of the solubility of the colloid. As a result of this reduction a large part of the colloid can be separated out into a new phase. The original one phase system becomes two phases. This process consists of three steps-

Formation of three immiscible phases; a liquid manufacturing phase, a core material phase and a coating material phase.

Deposition of the liquid polymer coating on the core material 
Rigidizing of the coating material

Step-1: The first step of coacervation phase separation involves the formation of three immiscible chemical phases: a liquid vehicle phase, a coating material phase and a core material phase. The three phases are formed by dispersing the core material in a solution of coating polymer, the vehicle phase is used as a solvent for polymer. The coating material phase consists of a polymer in a liquid phase, is formed by using one of the of phase separation- coacervation method, i.e. by changing the temperature of the polymer solution, by adding a solution, or by inducing a polymer- polymer interaction.

Step-2: It involves the deposition of the liquid polymer coating upon the core material. This is done by controlled mixing of liquid coating material and the core material in the manufacturing vehicle. The liquid coating polymer deposited on the core material if the polymer is adsorbed at the interface formed between the core material and liquid phase. The reduction in the total free interfacial energy of the system help to promote the deposition of the coating material, brought by the decrease of the coating material surface area during coalescence of the liquid polymer droplets.

Step-3: In the last step rigidizing of the coating material done by the thermal, cross linking desolvation techniques, to forms a self supporting microcap

\section{Simple Coacervation}

Simple coacervation involves the use of either a second more-water soluble polymer or an aqueous non-solvent for the gelatin. This produces the partial dehydration/desolvation of the gelatin molecules at a temperature above the gelling point. This results in the separation of a liquid gelatin-rich phase in association with an equilibrium liquid (gelatin-poor) which under optimum separation conditions can be almost completely devoid of gelatin. Simple coacervation can be effected either by mixing two colloidal dispersions, one having a high affinity for water, or it can be induced by adding a strongly hydrophilic substance such as alcohol or sodium sulfate. The water soluble polymer is concentrated in water by the action of a water miscible, non-solvent for the emerging polymer (gelatin) phase. Ethanol, acetone, dioxane, isopropanol and propanol have been used to cause separation of coacervate of gelatin, polyvinyl alcohol and methyl cellulose. Phase separation can be effected by the addition of an electrolyte such as an inorganic salt to an aqueous solution of a polymer such as gelatin, polyvinyl alcohol or carboxymethyl cellulose.

\section{Complex Coacervation}

Complex coacervation' can be induced in systems having two dispersed hydrophilic colloids of opposite electric charges. Neutralization of the overall positive charges on one of the colloids by the negative charge on the other is used to bring about separation of the polymer-rich complex coacervate phase. The gelatin-gum arabic (gum acacia) system is the most studied complex coacervation system. Complex coacervation is possible only at $\mathrm{pH}$ values below the isoelectric point of gelatin. It is at these $\mathrm{pH}$ values that gelatin becomes positively charged, but gum arabic continues to be negatively charged. A typical complex coacervation process using gelatin and gum arabic colloids is as follows: The core material is emulsified or suspended either in the gelatin or gum arabic solution. The aqueous solution of both the gelatin and gum arabic should each be below 3 percent by weight. Then, the gelatin or the gum 
arabic solution (whichever was not previously used to suspend the core material) is added into the system. The temperature of the system must be higher than the gel point of an aqueous gelatin solution (greater than $35^{\circ} \mathrm{C}$ ). The $\mathrm{pH}$ is adjusted to $3.8-4.3$ and continuous mixing is maintained throughout the whole process. The system is cooled to $50^{\circ} \mathrm{C}$ and the gelled coacervate capsule walls are insolubilized by either adding glutaraldehyde or another hardening agent or adjusting the $\mathrm{pH}$. The microcapsules are washed, dried and collected (Gunder et al., 1995; Ghulam Murtaza et al., 2009).

\section{Aqueous Phase Separation}

The term aqueous phase separation is often more simply described as "oil-in-water" microencapsulation. The two encapsulation processes described above are examples of this "oil-in-water" encapsulation. In this process the core material is the oil and it should be immiscible in the continuous phase, namely water.

\section{Organic Phase Separation}

The term organic phase separation' is sometimes more simply referred to as "water-in-oil" microencapsulation. In this case the polar core is dispersed into an oily or non-polar continuous medium. The wall material is then dissolved in this continuous medium.

A simple technique for encapsulation consists of dissolving ethyl cellulose in cyclohexane at a temperature of $50^{\circ} \mathrm{C}$ with continuing mixing. Only one phase is present. The cyclohexane is the oily, continuous phase and the ethyl cellulose will later form the coacervative wall. The temperature is elevated to $70^{\circ} \mathrm{C}$ over a period of 20 to 30 minutes. The core material is added and the temperature raised to $80^{\circ} \mathrm{C}$ over a period of time and is held at that temperature for one hour. The system is allowed to cool rapidly to $20-40^{\circ} \mathrm{C}$. Upon cooling, the ethyl cellulose will gradually emerge as a separated coacervate phase which will then gradually solidify by the time $20^{\circ} \mathrm{C}$ is reached (unlike hot cyclohexane, the cold material is a nonsolvent). The capsules are washed, filtered and air dried.

\section{Polymer Encapsulation by Rapid Expansion of Supercritical Fluids}

Supercritical fluids are highly compressed gasses that possess several advantageous properties of both liquids and gases. These fluids have attracted much attention in recent years, the most widely used being supercritical $\mathrm{CO} 2$, alkanes (C2 to $\mathrm{C} 4)$, and nitrous oxide (N2O). They have low hydrocarbon-like solubility for most solutes and are miscible with common gases such as hydrogen (H2) and nitrogen. A small change in temperature or pressure causes a large change in the density of supercritical fluids near the critical point a property which enhances their use in several industrial applications.

\section{The Most widely used Methods are as follows}

Rapid expansion of supercritical solution (RESS)

Gas anti-solvent (GAS)

Particles from gas-saturated solution (PGSS)

\section{Rapid Expansion of Supercritical Solution}

In this process, supercritical fluid containing the active ingredient and the shell material are maintained at high pressure and then released at atmospheric pressure through a 
small nozzle. The sudden drop in pressure causes dissolvation of the shell material, which is then deposited around the active ingredient (core) and forms a coating layer. The disadvantage of this process is that both the active in- gradient and the shell material must be very soluble in supercritical fluids.

\section{Gas Anti-solvent (GAS) Process}

This process is also called supercritical fluid anti-solvent (SAS). Here, supercritical fluid is added to a solution of shell material and the active ingredients and maintained at high pressure. This leads to a volume expansion of the solution that causes super saturationocapsule.

Such that precipitation of the solute occurs. Thus, the solute must be soluble in the liquid solvent, but should not dissolve in the mixture of solvent and supercritical fluid. On the other hand, the liquid solvent must be miscible with the supercritical fluid. This process is unsuitable for the encapsulation of water-soluble ingredients as water has low solubility in supercritical fluids. It is also possible to produce submicron particles using this method (Jegat et al., 2000).

\section{Particles from a Gas-saturated Solution (PGSS)}

This process is carried out by mixing core and shell materials in supercritical fluid at high pressure. During this process supercritical fluid penetrates the shell material, causing swelling. When the mixture is heated above the glass transition temperature the polymer liquefies.

Upon releasing the pressure, the shell material is allowed to deposit onto the active ingredient. In this process, the core and shell materials may not be soluble in the supercritical fluid.

\section{Centrifugal Extrusion}

Liquids are encapsulated using a rotating extrusion head containing concentric nozzles. In this process, a jet of core liquid is surrounded by a sheath of wall solution or melt. As the jet moves through the air it breaks, owing to Rayleigh instability, into droplets of core, each coated with the wall solution. While the droplets are in flight, a molten wall may be hardened or a solvent may be evaporated from the wall solution.

\section{Pan Coating}

The pan coating process, widely used in the pharmaceutical industry, is among the oldest industrial procedures for forming small, coated particles or tablets. The particles are tumbled in a pan or other device while the coating material is applied slowly. The pan coating process, widely used in the pharmaceutical industry, is among the oldest industrial procedures for forming small, coated particles or tablets. The particles are tumbled in a pan or other device while the coating material is applied slowly with respect to microencapsulation, solid particles greater than 600 microns in size are generally considered essential for effective coating, and the process has been extensively employed for the preparation of controlled - release beads. Medicaments are usually coated onto various spherical substrates such as nonpareil sugar seeds, and then coated with protective layers of various polymers.

\section{Spray-drying}

Spray drying serves as a microencapsulation technique when an active material is dissolved or suspended in a melt or polymer solution and becomes trapped in the dried particle. The main advantages is the ability to handle labile materials because of the 
short contact time in the dryer, in addition, the operation is economical. In modern spray dryers the viscosity of the solutions to be sprayed can be as high as $300 \mathrm{~m}$. The equipment components of a standard spray dryer include an air heater, atomizer, main spray chamber, blower or fan, cyclone and product collector. Microencapsulation by spray congealing can be accomplished with spray drying equipment when the protective coating is applied as a melt.

\section{Fluidized-bed Technology}

With the high demand for encapsulated materials in the global market, fluid-bed coaters have become more popular. They are used for encapsulating solid or porous particles with optimal heat exchange. The liquid coating is sprayed onto the particles and the rapid evaporation helps in the formation of an outer layer on the particles. The thickness and formulations of the coating can be obtained as desired.

\section{Chemical Method}

\section{Solvent Evaporation}

The processes are carried out in a liquid manufacturing vehicle. The microcapsule coating is dissolved in a volatile solvent, which is immiscible with the liquid manufacturing vehicle phase. A core material to be microencapsulated is dissolved or dispersed in the coating polymer solution. With agitation, the core coating material mixture is dispersed in the liquid manufacturing vehicle phase to obtain the appropriate size microcapsule. The mixture is then heated (if necessary) to evaporate the solvent for the polymer. In the case in which the core material is dispersed in the polymer solution, polymer shrinks around the core. In the case in which core material is dissolved in the coating polymer solution, a matrix - type microcapsule is formed. Once all the solvent for the polymer is evaporated, the liquid vehicle temperature is reduced to ambient temperature (if required) with continued agitation. At this stage, the microcapsules can be used in suspension form, coated on to substrates or isolated as powders.

\section{Polymerization}

In this technique the capsule shell will be formed at or on the surface of the droplet or particle by polymerization of the reactive monomers. The substances used are multifunctional monomers. Generally used monomers include multifunctional isocyanates and multifunctional acid chlorides. These will be used either individually or in combination. The multifunctional monomer dissolved in liquid core material and it will be dispersed in aqueous phase containing dispersing agent. A coreactant multifunctional amine will be added to the mixture. This results in rapid polymerization at interface and generation of capsule shell takes place. A poly urea shell will be formed when isocyanate reacts with amine, polynylon or polyamide shell will be formed when acid chloride reacts with amine. When isocyanate reacts with hydroxyl containing monomer produces polyurethane shell. Like IFP the capsule shell formation occurs because of polymerization monomers added to the encapsulation reactor.

\section{Interfacial Polymer}

In Interfacial polymerization, the two reactants in a polycondensation meet at an interface and react rapidly. The basis of this method is the classical Schotten Baumann reaction between an acid chloride and a compound containing an active hydrogen atom, such as an amine or alcohol, 
polyesters, polyurea, polyurethane. Under the right conditions, thin flexible walls form rapidly at the interface.

\section{In-situ Polymerization}

In a few microencapsulation processes, the direct polymerization of a single monomer is carried out on the particle surface. In one process, E.g. Cellulose fibers are encapsulated in polyethylene while immersed in dry toluene. Usual deposition rates are about $0.5 \mu \mathrm{m} / \mathrm{min}$. Coating thickness ranges $0.2-75 \mu \mathrm{m}$. The coating is uniform, even over sharp projections (Nikhil, 2005).

\section{Matrix Polymer}

In a number of processes, a core material is imbedded in a polymeric matrix during formation of the particles. A simple method of this type is spray-drying, in which the particle is formed by evaporation of the solvent from the matrix material. However, the solidification of the matrix also can be caused by a chemical change.

\section{Applications of Microencapsulation}

Some of the applications of microencapsulation are illustrated as below:

It has been used to protect drugs from environmental hazards such as humidity, light, oxygen or heat. Microencapsulation does not yet provide a perfect barrier for great materials, which degrade in the presence of oxygen, moisture or heat, however a degree of protection against these factors can be provided

The separations of incompatible substances. Microencapsulation can be used to decrease the volatility. An encapsulated volatile substance can be stored for longer times without substantial evaporation
Microencapsulation has also been used to decrease potential danger of handling of toxic or noxious substances. The toxicity occurred due to handling of fumigants, herbicides, insecticides and pesticides have been advantageously decreased after microencapsulation.

The hygroscopic properties of many core materials may be reduced by microencapsulation.

Many drugs have been microencapsulated to reduce.

\section{Current and Future Developments}

Micro encapsulation formulations have many advantages over normal formulations. With this formulation, less frequent drug administration is possible, lower plasma peak concentration can be obtained to avoid adverse effects. This technology to ensure time controlled pulsatile release of bioactive compounds has been developed (Goudanavar et al., 2010). Microencapsulated product increase the stability, bioavailability and dissolution of the drug, properties. This is also useful in the other fields like biotechnology for the cell immobilization, in food industry, in drug delivery etc (Goud et al., 2005). The pulsatile delivery of bioactive compound is possible. In case pharmaceuticals micro encapsulation could bring improvement of exciting approaches to treatment of various diseases. Still, numerous studies of micro encapsulation techniques are believed to result in the development of new and effective clinical protocols in near future. Various type of micro encapsulated drug delivery system have been studied in the recent year these system release the drug in respond to various physiological stimuli according to need of body. Thus it shows 
Int.J.Curr.Res.Aca.Rev.2016; 4(5): 186-196

broad potential opportunities in using resolving important clinical problems. various strategies of drug targeting for

Table.1 Various Microencapsulation Techniques and the Processes Involved in each Technique

\begin{tabular}{|c|c|c|}
\hline No. & $\begin{array}{l}\text { Microencapsulation } \\
\text { technique }\end{array}$ & Major steps in encapsulation \\
\hline 1 & Spray-drying & $\begin{array}{l}\text { a. Preparation of the dispersion } \\
\text { b. Homogenization of the dispersion } \\
\text { c. Atomization of the infeed dispersiond. } \\
\text { Dehydration of the atomized particles }\end{array}$ \\
\hline 2 & Spray-chilling & $\begin{array}{l}\text { a. Preparation of the dispersion } \\
\text { b. Homogenization of the dispersion } \\
\text { c. Atomization of the infeed dispersion }\end{array}$ \\
\hline 3 & Spray-cooling & $\begin{array}{l}\text { a. Preparation of the dispersion } \\
\text { b. Homogenization of the dispersion } \\
\text { c. Atomization of the infeed dispersion }\end{array}$ \\
\hline \multirow[t]{2}{*}{4} & A. Extrusion & $\begin{array}{l}\text { a. Preparation of molten coating } \\
\text { b. Dispersion of core into molten polymer } \\
\text { c. Cooling or passing of core-coat mixture } \\
\text { through dehydrating liquid }\end{array}$ \\
\hline & B. Centrifugal extrusion & $\begin{array}{llll}\text { a. Preparation of } & \text { core } & \text { solution } \\
\text { b. Preparation of coating material } & \text { solution } \\
\text { c. Co-extrusion of core and coat } & \text { solution } \\
\text { through nozzles }\end{array}$ \\
\hline 5 & Fluidized-bed coating & $\begin{array}{l}\text { a. Preparation of coating solution } \\
\text { b. Fluidization of core particles. } \\
\text { c. Coating of core particles }\end{array}$ \\
\hline 6 & Liposomal entrapment & $\begin{array}{l}\text { a. Microfluidization } \\
\text { b. Ultrasonication } \\
\text { c. Reverse-phase evaporation }\end{array}$ \\
\hline 7 & Lyophilization & $\begin{array}{l}\text { a. Mixing of core in a coating solution } \\
\text { b. Freeze-drying of the mixture }\end{array}$ \\
\hline 8 & Coacervation & $\begin{array}{l}\text { a. Formation of a three-immiscible chemical } \\
\text { phases } \\
\text { b. Deposition of the coating. Solidification of } \\
\text { the coating }\end{array}$ \\
\hline 9 & $\begin{array}{l}\text { Centrifugal suspension } \\
\text { separation }\end{array}$ & $\begin{array}{l}\text { a. Mixing of core in a coating material } \\
\text { b. Pour the mixture over a rotating disc to } \\
\text { obtain encapsulated tiny particles } \\
\text { c. Drying }\end{array}$ \\
\hline 10 & Cocrystallization & $\begin{array}{l}\text { a. Preparation of supersaturated sucrose } \\
\text { solution } \\
\text { b. Adding of core into supersaturated solution } \\
\text { c. Emission of substantial heat after solution } \\
\text { reaches the sucrosecrystallization } \\
\text { temperature }\end{array}$ \\
\hline 11 & Inclusion complexation & $\begin{array}{l}\text { Preparation of complexes by mixing or grinding } \\
\text { or spray-drying }\end{array}$ \\
\hline
\end{tabular}


Fig.1 Coacervation Process: (a) Core Material Dispersion in Solution of Shell Polymer; (b) Separation of cCoacervate from Solution; (c) Coating of Core Material by Micro Droplets of Coacervate; (d) Coalescence of Coacervate to form Continuous Shell Around Core

Particles

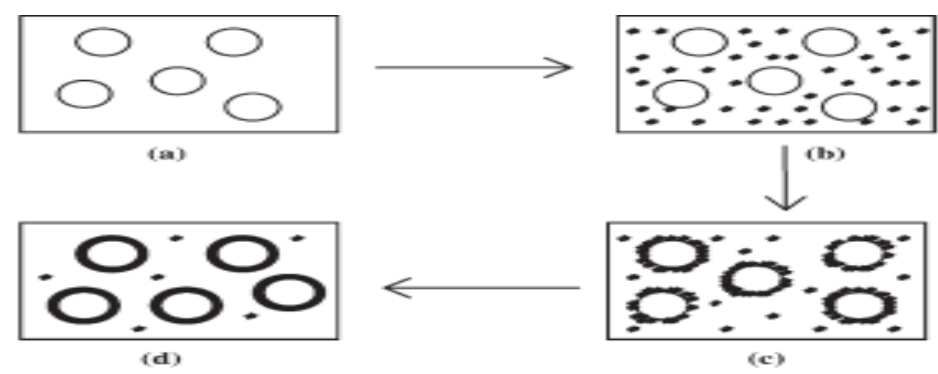

Fig.2 Microencapsulation by Rapid Expansion of Supercritical Solutions

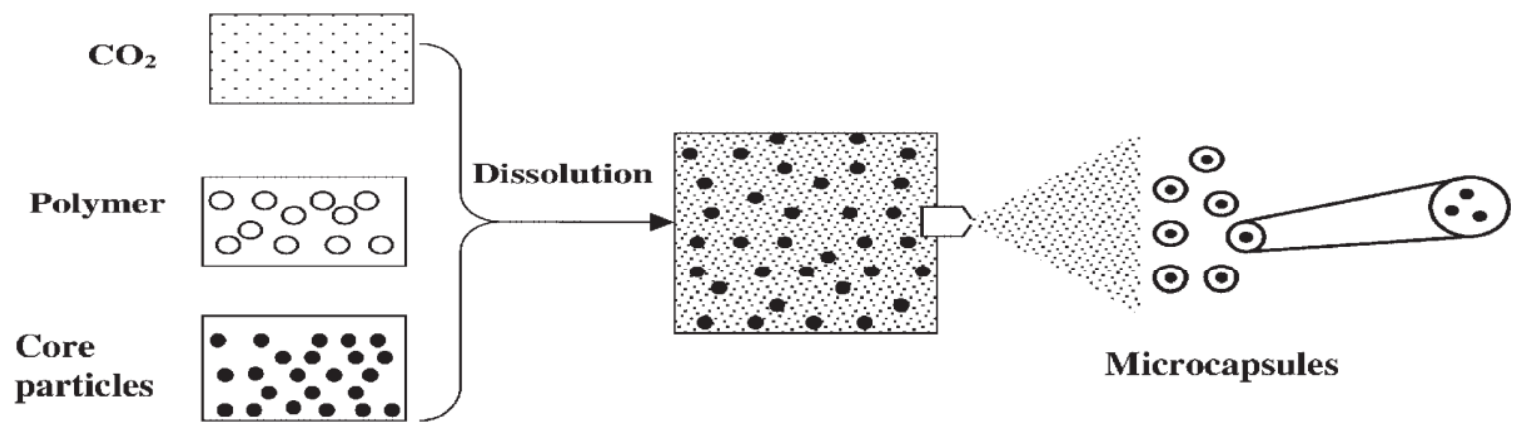

\section{Conclusion}

The research in the area of microencapsulation has huge potential to give raw material advantageous traits resulting in superior products. The very much popular microencapsulation technique is the most convenient way of protection and masking, reduce dissolution rate, facilitation of handling, and spatial targeting of active ingredient. Although significant advantage have been made in the field of microencapsulation, still many challenges need to be rectified during the appropriate selection of core materials, coating materials and process techniques. The microencapsulation approach also beneficial for those drugs which required to dissolved into the intestine not in the stomach. Therefore, this safe and efficient particular system should be developed in future.

\section{References}

Tarcha, P.J. 1991. Polymers for controlled drug delivery. Boca Raton: $C R C$, Meier W.

Chemical Society Reviews. 2000. 29(5): 295-303.

Mo“hwald, H., Donath, E., Sukhorukov, G. 2003. Smart capsules. New York: Wiley VCH.

Peyratout, C.S. 2004. Da“hne L. Angewandte Chemie International Edition, 43: 3762e83. 
Discher, B.M., Won, Y.Y., Ege, D.S., Lee, J.C.M., Bates, F.S., Discher, D.E., et al. Sci., 284: 1143e 6.

Dinsmore, A.D., Hsu, M.F., Nikolaides, M.G., Marquez, M., Bausch, A.R., Weitz, D.A. Sci., 98(5595): 1006e9.

Brazel, S.C., Peppas, N.A. 2000. Modeling of drug release from swellable polymers. Eur. J. Pharm. Biopharm., 49: 47-48.

Korsmeyer, R.W., Gurny, R., Doelker, E.M., Buri, P., Peppas, N.A. 1983. Mechanism of solute releas from porous hydrophilic polymers. Int. J. Pharm., 15(1): 25-35.

Gunder, W., Lippold, B.H., Lippold, B.C. 1995. Release of drugs from ethyl cellulose microcapsules (diffusion pellets) with pore formers and pore fusion. Euro. J. Pharm. Sci., 3: 20314.

Costa, P., Lobo, J.M.S. 2001. Modeling and comparison of dissolution profiles. Eur. J. Pharm. Sci., 13: 123-33.

Lachman, L., Lieberman, H.A., Kanig, J.L., editors. The theory and practice of industrial pharmacy. 3rd ed. Ch. 13,

Thies, C., Bissey, M.C. 1983. Biomedical applications of micro encapsulation.

Florida: CRS Press. Part III. 1991, Varghese Publishing House, Bombay 412-28.

Guo, J.H. 1994. Bioadhesive polymer buccal patches for buprenorphine controlled delivery: formulation in vitro adhesive and release properties. Drug Dev. Ind. Pharm., 20: 315-325.
Gunder, W., Lippold, B.H., Lippold, B.C. 1995. Release of drugs from ethyl cellulose microcapsules (diffusion pellets) with pore formers and pore fusion. Euro. J. Pharm. Sci., 3: 203-214.

Ghulam Murtaza, Mahmood Ahamd, Naveed Akhtar, Fatima Rasool. 2009. A

comparative study of various microencapsulation techniques: effect of polymer viscosity on microcapsule characteristics. Pak. J. Pharm. Sci., 3: 291-300.

Jegat, C., Taverdet, J.L. 2000. Stirring speed influence study on microencapsulation process and the drug release from microcapsules. Polymer Bull., 44: 345-351.

Korsmeye, R.W., Gurny, R., Doelker, E.M., Buri, P., Peppas, N.A. 1983. Mechanism of solute release from porous hydrophilic polymers. Int. J. Pharmacet., 15: 25-35.

Nikhil, K., Sachan. 2005. Controlled drug delivery through microencapsulation. Assam India, Dibrugarh University.

Goudanavar, P.S., et al. 2010. "Design and characterasation of sustained Release Microcaplsules of Salbutamol Sulphate" Int. J. Pharm. Tech. Res., Vol 2, No. 2, P. No. 1144-1149.

Goud, K., Park, H.J. 2005. Recent Developments in Microencapsulation of Food Ingredients. Drying Technol., 23: 1361-1394.

\section{How to cite this article:}

Sonal Salve and Vaibhav Changediya. 2016. Microcapsule: A Dosage Form for Drug Delivery in Novel Drug Delivery System. Int.J.Curr.Res.Aca.Rev.4(5): 186-196.

doi: http://dx.doi.org/10.20546/ijcrar.2016.405.019 